\title{
La educación estadística en el nivel universitario: retos y oportunidades
}

\author{
Luis Fernando Ramos Vargas* \\ Universidad Católica de Santa María, Arequipa, Perú \\ https://orcid.org/0000-0001-9216-7040
}

\begin{abstract}
Resumen
La educación estadística es un fenómeno que en los últimos años ha generado mayor interés, evidenciado en el incremento del número de investigaciones en todos los niveles educativos. El desarrollo tecnológico ha traído consigo cambios importantes en las sociedades, y ha propiciado una inmensa cantidad de información que crece diariamente, por lo que se necesita a otro tipo de ciudadano y profesional, que sea capaz de entender e interpretar la información que lo rodea. Sin embargo, los estudiantes universitarios muestran actitudes desfavorables hacia la estadística, lo que se ve reflejado en resultados insatisfactorios en término de aprendizaje y aplicación. Se trata de una investigación bibliográfica-documental que revisa los factores implicados en los procesos de enseñanza y aprendizaje de la estadística en el nivel universitario. La exposición se organiza en cuatro partes, en la primera, se presenta la problemática de la educación estadística en las aulas universitarias; en la segunda, se explican los niveles cognitivos involucrados; en la tercera, se exponen siete modelos de enseñanza y en la última se discuten las implicancias del futuro de la educación estadística en el nivel universitario. Palabras clave: didáctica de la estadística, enseñanza superior, educación estadística.
\end{abstract}

\section{Statistics Training at the University Level: Challenges and Opportunities}

\begin{abstract}
The training on statistics is a phenomenon that has sparked great interest in recent years, which is evidenced by the increase in the number of research work at all educational levels. Technological development has brought with it important changes to society and has produced an immense amount of information that grows daily, which is why a different type of citizen and professional is needed; one who is able to understand and interpret the information that surrounds him or her. However, university students show unfavorable attitudes towards statistics, which can be seen in the unsatisfactory results in terms of learning and application. This is a bibliographic-documentary research that examines the elements that take part in the statistical teaching and learning processes at the university level. The exhibition is organized in four parts: the first presents the problems of statistical education in university classrooms; the second explains the cognitive levels involved; the third presents seven teaching models; and the last one examines the implications for the future of statistical education at the university level.
\end{abstract}

Keywords: didactics of statistics, higher education, statistical education 


\title{
A educação estatística no âmbito universitário: desafios e oportunidades
}

\begin{abstract}
Resumo
A educação estatística é um fenómeno que nos últimos anos tem gerado muito interesse, evidenciado no aumento do número de pesquisas em todos os níveis educativos. O desenvolvimento tecnológico trouxe consigo mudanças importantes nas sociedades, e tem propiciado uma imensa quantidade de informação que cresce diariamente. Portanto, é preciso outro tipo de cidadão e profissional que seja capaz de entender e interpretar a informação que o rodeia. No entanto, os estudantes universitários mostram atitudes desfavoráveis para com a estatística, fato refletido nos resultados insatisfatórios em termos de aprendizagem e aplicação. Trata-se de uma pesquisa bibliográfica-documental que revisa os fatores implicados nos processos de ensino e aprendizagem da estatística no âmbito universitário. A exposição organiza-se em quatro partes; na primeira, apresenta-se a problemática da educação estatística nas salas de aula universitárias; na segunda, explicam-se os níveis cognitivos envolvidos; na terceira, expõem-se sete modelos de ensino e na última discutem-se as implicâncias do futuro da educação estatística no âmbito universitário.
\end{abstract}

Palavras-chaves: didática da estatística, ensino superior, educação estatística.

Como citar:

Ramos, L. (2019). La educación estadística en el nivel universitario: retos y oportunidades. Revista Digital de Investigación en Docencia Universitaria, 13(2), 67- 82. http://doi.org/10.19083/ridu.2019.1081

E n la actualidad, el desarrollo de la sociedad de la información, el auge de la tecnología, y la necesidad de interpretar la abundante información disponible, ha propiciado que la estadística cobre mayor importancia (Ruiz, 2015) en la participación ciudadana y la toma de decisiones personales y laborales. Esta abundante información implica una mayor necesidad de desarrollar competencias estadísticas en los estudiantes de todo nivel, y especialmente los estudiantes universitarios (Blanco, 2018).

Salinas y Mayén (2016) afirman que el aprender estadística se ha vuelto algo indispensable para el ciudadano, que le permite analizar, interpretar y tomar decisiones en base a la información disponible. En este sentido, Zapata (2011) afirma que "el ciudadano común enfrenta el desafío permanente de leer e interpretar datos estadísticos que surgen de diferentes fuentes. Infortunadamente, nuestros ciudadanos cuentan con una alfabetización estadística insuficiente para enfrentar con éxito estos retos que la cultura demanda" (p. 234).

Esta alfabetización estadística se constituye como un elemento fundamental en la sociedad moderna, ya que los ciudadanos necesitan formación estadística para comprender el espacio en el que se desarrollan, esto les permitirá evaluar críticamente los datos en contextos sociales y les brindará la habilidad de tomar decisiones informadas. El ser un ciudadano estadísticamente alfabetizado se ha vuelto necesario para todos, independientemente de la profesión, clase social o nivel educativo, convirtiendo a la estadística en una parte importante de la educación general deseable en todo ciudadano (Sanoja \& Ortíz, 2013).

La educación estadística se le considera como una disciplina emergente en continua consolidación y expansión (Andrade, Fernández, \& Alfonso, 2017), que se encuentra dando avances importantes, incluso mayor al de otras ramas de matemática, en lo concerniente a su extensión como a su profundidad (Batanero, 2018). Su producción se incrementa alrededor del mundo, y en todos los niveles educativos, en donde se observa su incorporación en el currículo escolar de diversos países, a nivel primario (Ruiz, 2015; Alsina, 2017), secundario y superior (Toapanta-Toapanta, Pérez-Narváez, \& Lema-Yungan, 2018), y en la mayo- 
ría de las carreras universitarias (Comas, Martins, Nascimento, \& Estrada, 2017), desarrollándose de esa manera, una cultura estadística (Behar, Grima, Ojeda, \& Cruz, 2013).

Según lo expuesto, se entiende que la asignatura de estadística comprende gran importancia en el desarrollo profesional y ciudadano. Sin embargo, muchas veces esta asignatura es percibida de forma aversiva por el estudiante, incluso a pesar de reconocer la importancia dentro de su formación.

Behar y Grima (2004) refieren que, al hablar de la problemática en la enseñanza de la estadística, se debe considerar: los niveles altos de ansiedad que genera, las actitudes negativas hacia esta, el contenido del curso (excesivamente centrado en probabilidad y matemáticas) que muestra poca relación con el mundo real, la falta de motivación de los estudiantes, las frustraciones de algunos profesores por no ver resultados a sus esfuerzos, la influencia de la tecnología en el aprendizaje y si es que realmente los profesores tienen claro lo que se quiere de los estudiantes respecto a la educación estadística. Las asignaturas de estadística se han considerado como los cursos más temidos y hasta, podría ser, el curso que genera mayor ansiedad en el estudiante universitario y de post-grado (Onwuegbuzie, 2004), especialmente en carreras relacionadas a las ciencias sociales (Paechter, Macher, Martsvishvili, Wimmer, \& Papousek, 2017), e incluso se le ha considerado por los estudiantes como la asignatura más temida de la malla curricular (Molina, Rodrigo, \& Bonavia, 2011). Esta situación ha propiciado que se formulen términos propios al estudiar estos fenómenos, por ejemplo: la ansiedad estadística, frecuentemente encontrado en la literatura científica (Mason \& Reid, 2018), y el término Statisticophobia (Dillon, 1992) que se refiere a tener miedo hacia la estadística.

Se ha documentado una fuerte relación entre las actitudes de los estudiantes hacia la estadística y sus resultados en la asignatura (Smith, 2017). Comas et al. (2017) explican que "las actitudes son parte integrante de todas las materias de aprendizaje y ocupan un lugar central en el acto educativo, guiando el proceso perceptivo y cognitivo que comparta el aprendizaje de cualquier contenido educativo" (p.480). Para un gran número de estudiantes universitarios, las asignaturas de estadística implican una contradicción cognitiva y afec- tiva sobre el proceso de enseñanza y aprendizaje, ya que el estudiante entiende que la estadística es una ciencia muy importante que sirve para analizar datos en investigaciones científicas, pero, por otro lado, la asignatura les resulta tediosa, aburrida o inclusive es percibida como fuente de ansiedad, es más si es que pudieran hacerlo, posiblemente la evitarían. Desde un punto de vista de las actitudes hacia la estadística, este conflicto entre la dimensión cognitiva y afectiva influye sobre una tercera dimensión que es la dimensión conductual, es decir, que intelectualmente reconocen su importancia, pero afectiva y conductualmente no están inclinados a utilizarla. Pérez, Aparicio, Bazán y Abdounur (2015) realizaron una investigación sobre las actitudes hacia la estadística en estudiantes universitarios colombianos y encontraron que los estudiantes reconocen la importancia de las estadísticas, pero muestran desconfianza sobre su uso, la capacidad requerida y el gusto por la disciplina.

Son muchos los estudiantes que llegan a cursar esta asignatura con actitudes desfavorables. Es necesario que los docentes del curso estén preparados para trabajar sobre estas actitudes y modificarlas. Pero el reto es aún mayor, ya que muchos de los docentes no presentan estas capacidades, ya sea por desconocimiento de modelos de enseñanza que favorezcan el aprendizaje de la estadística o porque no poseen actitudes favorables hacia la asignatura, lo que implica que estas actitudes negativas puedan ser transmitidas dentro de lo que se conoce como currículo oculto. Tarazona, Bazán y Aparicio (2013) afirman que los maestros pueden transmitir actitudes positivas y negativas de manera inconsciente a sus alumnos y esto puede afectar en su aprendizaje. Si consideramos la poca preparación que tienen algunos docentes para la enseñanza del curso, el panorama se vuelve más complicado. Al respecto, Estrella (2017) explica que la escasa preparación en la enseñanza y aprendizaje de la estadística que tienen los profesores en formación y en ejercicio, es un problema que se genera al intentar promover y mejorar la capacidad de los estudiantes a pensar estadísticamente.

Atendiendo a esta problemática de la enseñanza de la estadística, se elabora el presente artículo de revisión, que tiene el objetivo de analizar los factores implicados en los procesos de enseñanza que im- 
pactan en el aprendizaje de la estadística en el contexto universitario. Se trata de una investigación bibliográfica documental. Los temas abordados se presentan en el siguiente orden: en primer lugar, se analiza la formación estadística en la universidad; en segundo lugar, se presentan los niveles cognitivos propuestos para los procesos de enseñanza-aprendizaje de la asignatura; en tercer lugar, se describen siete modelos didácticos sobre la enseñanza del curso y finalmente se discuten implicancias sobre el futuro de la educación estadística.

\section{Formación Estadística en el Nivel Universitario}

En muchas aulas universitarias todavía se mantienen modelos tradicionales de educación que se centran excesivamente en la impartición de contenidos y dejando en segundo lugar a los estudiantes y, cómo es que estos logran sus aprendizajes. Dichos modelos han mostrado poca efectividad al momento de ser evaluados (en términos de aprendizaje de los estudiantes), por lo que en la actualidad han surgido nuevos que se centran más en el proceso y cómo es que este puede mejorarse.

La estadística es una de las asignaturas que tiene mayor presencia en las mallas curriculares de todas las carreras universitarias. Sin embargo, y como se comentó anteriormente, sucede un fenómeno peculiar, a pesar de reconocer que la estadística es importante para el desarrollo de la ciencia y de la sociedad; los docentes de la asignatura de estadística, principalmente de carreras como psicología, sociología, educación, ciencias de la comunicación, etc., ven que sus estudiantes presentan una actitud desfavorable ante el aprendizaje del curso (Rodríguez, 2011), incluso presentan esta actitud antes de haber llevado algún curso de estadística (Tarazona et al., 2013).

En carreras de letras, los estudiantes que comienzan a cursar asignaturas de estadística no tienen una base matemática sólida y frecuentemente evitan las actividades que comprendan números, e incluso la amenaza de trabajar con números puede ser suficiente motivo para que el estudiante abandone o cambie de curso (Dempster \& McCorry, 2009). Considerando esta predisposición negativa hacia la estadística, es recomendable que los docentes realicen evaluaciones a priori de las actitudes hacia la asignatura. Esto resulta importante, ya que la actitud comprende un carácter evaluativo, mediador entre el objeto y el sujeto que realiza, y que esta evaluación influye inherentemente como una predisposición a responder de determinada manera. Si el docente de la asignatura se orienta hacia la modificación de actitudes, tendrá mayores posibilidades de que el estudiante logre el aprendizaje, en el sentido que utilice la estadística fuera del salón de clases.

Posiblemente esta predisposición negativa hacia la asignatura se deba a experiencias pasadas que conllevaron emociones displacenteras asociadas con asignaturas que comprendan números, como lo son las matemáticas y la física. Ruiz de Miguel (2015) realizó un estudio no experimental con diseño ex post facto sobre las actitudes hacia la estadística, encontrando que es abordada por los estudiantes con cierto recelo y que sus actitudes varían en función de sus experiencias previas. Dempster y McCorry (2009) estudiaron la relación entre las experiencias previas en matemática, estadística e informática en 103 estudiantes universitarios de pregrado de psicología cuya edad promedio fue 19.34(2.64) años, y de los cuales el 78\% son mujeres, los cuales respondieron la Escala de Actitudes hacia la Estadística (SATS-36), y realizaron una evaluación del curso. Con ello, encontraron que el mejor predictor para las actitudes fueron las percepciones de sus habilidades matemáticas, al menos en el inicio de la asignatura, y que, al finalizarla, los mejores predictores fueron los propios conocimientos y las habilidades desarrolladas. Praetcher et al. (2017) explican que el éxito en el aprendizaje de la estadística está sujeta al previo éxito en las matemáticas durante el tiempo de colegio. Sin embargo, García Martínez, Fallas-Vargas y Romero-Hernández (2015) encontraron que los estudiantes que afirman haber recibido estadística dentro de los contenidos de otra asignatura tienen peores actitudes que aquellos que no han recibido dicha formación, lo que indica la importancia de la vinculación de contenidos y asignaturas en la formación estadística del estudiante. Aunque el presente trabajo no abordará con amplitud la aparición de las evaluaciones negativas hacia la asignatura, se recomienda al lector que se remita a los textos de Behar, Grima, Ojeda y Cruz (2013), Bertorello, Albrecth y Tauber (2010) y Blanco (2008) 
para una mayor comprensión del tema.

La esencia de lo expuesto recae en que el estudiante conoce que la formación estadística es importante, pero ellos (e incluso, en ocasiones, el mismo docente) presentan reacciones emocionales, actitudes y creencias negativas hacia esta materia ocasionando un escaso interés por su aprendizaje (Blanco, 2008). Tomando esta aseveración como punto de partida, cabe preguntarse qué acciones debe realizarse para el desarrollo de las competencias requeridas y que estas, después de cursar la asignatura, no queden solo en un silabo impresas sin ajustarse a la realidad.

En la actualidad, la educación estadística universitaria está evolucionando drásticamente, de modelos que se centraban en la sola impartición de conocimientos hasta modelos que cuestionan que temas incluir y como enseñar al estudiante. Los esfuerzos por entender este fenómeno han sido y son diversos, algunos apuntan a mejorar la experiencia del estudiante y del docente a través de mostrar su utilidad como pilar del método científico, también se ha señalado la importancia del uso de metáforas para mejorar su aprendizaje (Thomas, 2007), la planificación de clases teórico-prácticas con la implementación de la lectura e interpretación de artículos científicos con contenido estadístico (Díaz-Reissner \& Quintana-Molinas, 2018), mnemotécnicas para la retención y recuperación de información estadística (Lesser, 2011), la medición de las actitudes hacia la estadística (Bertollero et al., 2010; Blanco, 2008; Estrada, 2002), metodologías activas y de colaboración entre estudiantes, incorporando nuevas tecnologías y el empleo de ejemplos y datos empíricos reales para ilustrar sus conceptos metodológicos (Molina et al., 2011), la modificación de la estructura del curso para disminuir los niveles de ansiedad (Mason \& Reid, 2018), la estrategia JAPEST, que fomenta el trabajo grupal y la resolución de problemas reales (Acosta \& Mejía, 2017), la enseñanza problémica con el empleo de las Tecnologías de la Información y las Comunicaciones (Vázquez, Aguilar, Chávez, Bony, \& Montes de Oca, 2016), y en los últimos años se han encontrado resultados alentadores que emplean el aprendizaje gamificado para la modificación de actitudes hacia la estadística (Smith, 2017). Estrella (2017) afirma que "en este escenario desafiante y dinámico, la Didáctica de la Estadística (Educación Estadística) se ha convertido en un creciente y apasionante campo de investigación y desarrollo" (p. 174).

El deseo de mejorar en la enseñanza de la estadística ha propiciado un gran conjunto de información sobre cómo mejorar el proceso enseñanza-aprendizaje de la asignatura, y es en este campo del conocimiento, donde se desea dirigir este texto y motivar a los profesores y estudiantes sobre el aprovechamiento de la estadística como pilar del conocimiento y pieza clave en el método científico. Antes de desarrollar los modelos didácticos de la estadística es importante definir con claridad la estructura jerárquica teorizada para su enseñanza.

\section{Niveles Cognitivos en el Aprendizaje de la Estadística}

Desde hace dos décadas, la investigación sobre la educación estadística se ha centrado en tres términos: la alfabetización (también llamada cultura estadística), el pensamiento y el razonamiento estadístico (Sánchez \& Berenguer, 2014). Campos (2016) considera a estos tres conceptos como competencias necesarias para la educación estadística, y agrega una competencia adicional que la denomina competencia crítica para la formación de la Educación Estadística Crítica. Estas competencias son importantes cuando se desean formular objetivos de aprendizaje, ya que estos términos dirigen la asignatura a través del diseño de actividades instruccionales, y la evaluación con instrumentos pertinentes. Sin embargo, estos conceptos se suelen superponer entre sí, lo que dificulta su correcto uso (Ben-Zvi \& Garfield, 2004).

Schield (2017) realiza una revisión conceptual del término alfabetización estadística y encuentra que existen diversas definiciones, lo que interpreta como una falta de consenso en la definición del término. De las definiciones revisadas en su artículo, se presenta la elaborada por la Asociación Estadística Americana (ASA) en su Informe GAISE (Guidelines for Assesment and Instruction in Statistics, 2005) que define a la alfabetización estadística como el entendimiento de su lenguaje básico (conceptos y símbolos) y las ideas fundamentales de la estadística.

Uno de los artículos más importantes sobre la definición de estos tres términos es discutido en el trabajo de Ben-Zvi y Garfield (2004), quienes 
afirman que los tres conceptos mencionados suelen confundirse frecuentemente, y ellos proponen definiciones específicas para identificar y diferenciar cada término señalado. Estas son:

- Alfabetización estadística: Comprende las habilidades básicas (organizar datos, elaborar tablas y trabajar con diferentes representaciones de los datos) empleadas para entender información estadística o los resultados de una investigación.

- Razonamiento estadístico: Definido como la manera en que la persona razona con ideas estadísticas y le da sentido a la información estadística.

- Pensamiento estadístico: Implica la comprensión del porque y como se conducen las investigaciones estadísticas y las ideas que subyacen a estas investigaciones, como: el concepto de la variabilidad, utilizar métodos adecuados acorde a los datos, presentación visual, muestreo, los modelos estadísticos. El pensamiento estadístico también se refiere a entender y utilizar el contexto de un problema para formular una investigación y ser capaz de criticar y evaluar los resultados de otras investigaciones.

Como se puede apreciar, la alfabetización estadística se encuentra en un nivel inferior, que se espera haber obtenido durante la etapa escolar, mientras que el pensamiento estadístico se espera consolidar en la etapa de educación superior. Régnier y Kuznetsova (2014) explican que el mayor objetivo de la educación estadística consiste en la formación del pensamiento estadístico. Para la reflexión, cabe señalar y cuestionarse si es que los estudiantes universitarios realmente han conseguido alcanzar el primer nivel (alfabetización estadística) o en todo caso, sería necesario tomar las medidas adecuadas (desde la alfabetización hacia el pensamiento estadístico) para alcanzar el nivel cognitivo superior.

\section{Modelos para la Enseñanza de la Estadística}

La enseñanza de la estadística conlleva estudiar un fenómeno con numerosas aristas, entre las que destacan: aspectos epistemológicos y psicológicos; los objetivos trazados a corto, mediano y largo plazo; la selección del contenido estadístico y los métodos de enseñanza; las alternativas para garantizar la motivación de los estudiantes; diversas metodologías para mejorar los resultados, el papel de la tecnología, los problemas estadísticos profesionales y los errores más comunes al aplicar metodología estadística (Sánchez \& Berenguer, 2014).

Estrella (2017) refiere que la Didáctica de la Estadística se ha convertido en un amplio campo de investigación y desarrollo. Además, afirma que este campo emergente, brinda resultados sobre cómo pueden ser utilizados en aulas escolares, y extendiéndose a niveles superiores como el universitario, para promover aprendizajes funcionales, profundos y duraderos. Asimismo, sostiene que existe una gran cantidad de investigación sobre la didáctica de la estadística, lo que ha favorecido a un cambio en el paradigma en la conceptualización de su enseñanza. Blanco (2018) explica que la evolución importante de esta nueva disciplina, en los últimos 10 años, plantea retos específicos en el ámbito de la docencia universitaria.

Este apartado del artículo toma como base lo desarrollado por Estrella (2017) y se orienta principalmente hacia el ámbito universitario. Para describir los modelos elegidos, es preciso definir qué se entiende por modelo para la enseñanza.

Witusba (2014) señala que los modelos de enseñanza de la estadística se emplean por motivos pedagógicos con la finalidad de acercar a los estudiantes a los conocimientos estadísticos de forma mucho más práctica, logrando que lo que se aprenda se replique. Para Orozco, Sosa y Martínez (2018), un modelo didáctico se refiere a "los planes estructurados que pueden usarse para configurar un currículo, diseñar materiales y orientar la enseñanza en las aulas" (p. 447). Entonces, un modelo para la enseñanza comprende una estrategia configurada por el docente para incrementar la efectividad del proceso enseñanza aprendizaje, mediante la modificación del contenido y material empleado en el aula, logrando la replicación de lo aprendido, en contextos que se encuentren tanto dentro como fuera del aula.

A continuación, se presentan siete modelos para la enseñanza de la estadística. Al momento de leer los modelos presentados, tenga presente que estos no son mutuamente excluyentes y pueden trabajarse simultánea o individualmente dependiendo de los requerimientos propios de la asignatura y del contexto en el que se dicta el curso. 


\section{Enseñar estadística: hacerlo memorable.}

Si bien Sowey (1995) no propone de forma estricta un modelo didáctico, sino que presenta un conjunto de sugerencias para mejorar el proceso de enseñanza aprendizaje, resulta importante incluirlo debido a que comprende conceptos interesantes que se abordarán también en los siguientes modelos revisados. Sowey (1995) escribe un artículo bien interesante sobre la enseñanza de la estadística denominado Enseñando estadística: Hacerlo memorable (Teaching Statistics: Making it Memorable). Aunque ya han pasado más de 20 años de la publicación de dicho artículo, es relevante presentarlo porque al momento de publicarse se trataba de una época distinta a la actual, en lo referente al uso de tecnología. Sowey, en su rol de profesor de estadística por más de 20 años en nivel universitario, se cuestiona sobre la naturaleza del aprendizaje y se formula a sí mismo la pregunta ¿Qué hace que la enseñanza sea memorable? y basado en su experiencia docente responde que dos aspectos son los más importantes: el sentido de estructura y el sentido de valor que tiene la asignatura. La primera dimensión, denominada sentido de estructura comprende dos subdimensiones: Coherencia, que se refiere a la explicación organizada de los temas y la integración de la estadística con la disciplina; y perspectiva, que comprende la construcción progresiva de conocimientos. En la segunda dimensión, llamada sentido de valor, comprende dos subdimensiones: el interés intelectual, que se refiere al contagio del interés del docente hacia el discente mediante la utilización de demostraciones para despertar la curiosidad y promover la reflexión; y resiliencia, que abarca a la capacidad del estudiante para afrontar retos que se constituye como un catalizador para el aprendizaje a largo plazo. Para profundizar sobre el tema, se recomienda la lectura de Sowey (1995).

\section{Modelo PPDAC.}

Wild y Pfannkuch (1999) proponen un modelo didáctico basado en la solución de problemas de investigación (desde la formulación hasta la conclusión) que comprende cuatro dimensiones: el ciclo investigativo (dimensión 1), tipos de pensamiento (dimensión 2), ciclo de preguntas (dimensión 3) y las disposiciones (dimensión 4). Este modelo fue producto de una serie de entrevistas a estudiantes de psicología y estadísticos profesionales quienes aportaron a formular un marco de trabajo para describir los procesos comprendidos en el razonamiento estadístico. Estrella (2017) explica que el ciclo PPDAC (que sus iniciales significan: Problema, Plan, Datos, Análisis y Conclusión) proporciona un marco de modelización de problemas estadísticos, gracias a que atiende a situaciones problemáticas reales (adaptables a cualquier ciencia) a través de la aplicación de la estadística. En la Figura 1 se observa un resumen del Ciclo de Investigación PPDAC propuesto por Wild y Pfannkuch (1999) y se han añadido aportes de Estrella (2017).

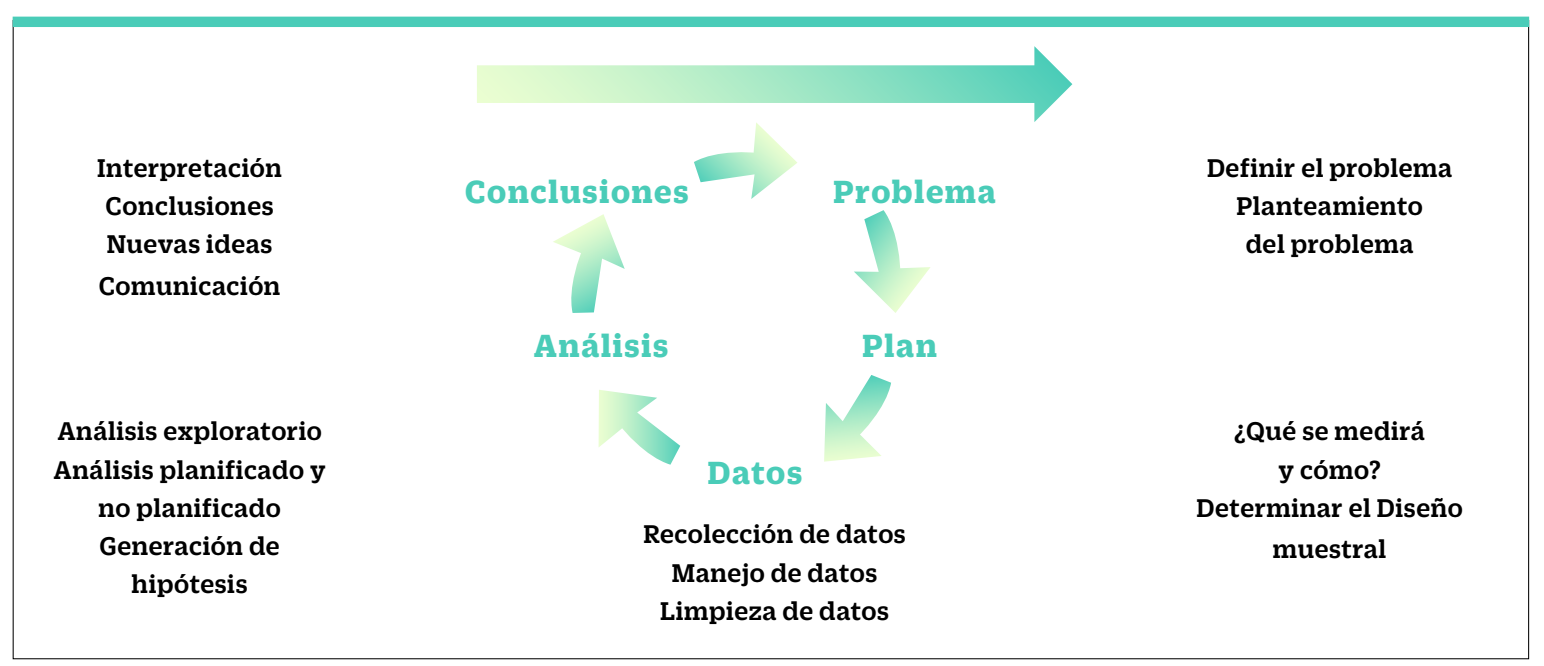

Figura 1. Ciclo investigativo PPDAC 


\section{Guía para la evaluación e Instrucción en Educación estadística (GAISE).}

Blanco (2018) afirma que desde los inicios el siglo XXI, la Asociación Estadística Americana (ASA) promueve y financia un conjunto de directrices para la enseñanza de estadística, que lo denominó Guidelines for Assesment and Introduction in Statistics (GAISE), teniendo como resultado un informe publicado en el año 2005 referido a la enseñanza introductoria de estadística en el nivel universitario. En el año 2016, estas directrices han sido actualizadas y "constituyen un buen resumen de cuál es la visión de la ASA acerca de lo que debería constituir una introducción a la Estadística hoy" (p. 255).

Schield (2017) realiza una revisión de este informe y encuentra que en la versión del año 2005 se tuvo como objetivo principal la alfabetización estadística, pero que en la revisión del año 2016 se reemplaza a la alfabetización estadística por el pensamiento estadístico como objetivo principal, evidenciando cambios en la primera recomendación de la versión 2005 que decía "enfatizar la alfabetización estadística y desarrollar el pensamiento científico" por "enfatizar el pensamiento científico" en la versión revisada del 2016. Aunque en el mismo trabajo se concluye que primero se requiere alcanzar la alfabetización estadística para seguir hacia el razonamiento y pensamiento estadístico. Estrella (2017) afirma que el informe GAISE proporcionó un marco que comprende cuatro componentes principales: formular preguntas, recopilar datos, analizar datos e interpretar resultados. Estas etapas son similares a las vistas en el Modelo PPDAC, salvo el segundo punto de este modelo que incluye una etapa específica para la planeación.

Este informe está dividido en dos partes: la primera enfatiza en la formación estadística en instituciones educativas de nivel básico y la segunda se centra en la formación estadística en Universidades. Ambos informes se pueden descargar de forma gratuita en la página web de la ASA. Como este artículo aborda la problemática en estudiantes universitarios, solo se tratará la información pertinente al caso.

La ASA (2016) señala las siguientes recomendaciones para la enseñanza introductoria en el nivel universitario: 1. Enseñar el pensamiento estadístico; 2. Enfocarse en la comprensión conceptual; 3. Integrar datos reales con un contexto y con un propósito; 4. Fomentar el aprendizaje activo; 5. Emplear la tecnología para explorar los conceptos y analizar los datos; 6. Utilizar evaluaciones para mejorar y evaluar el aprendizaje de los estudiantes.

La ASA (2016) explica que para alcanzar estas recomendaciones se requiere que el estudiante domine técnicas estadísticas que faciliten este entendimiento de los conceptos estadísticos y de los principios que subyacen a estas técnicas, por lo que no recomiendan introducir tópicos concretos que deban enseñarse en el curso. Blanco (2018) afirma que "el informe también señala algunos temas que podrían ser omitidos en un curso introductorio: teoría de la probabilidad, construcción manual de gráficos y Estadística Descriptiva Bási$\mathrm{ca}$, uso de tablas estadísticas y formación avanzada en programas de análisis estadístico" (p. 258).

Inferencia estadística informal (ISI). Rodríguez (2012) explica que, en el nivel universitario, todo curso introductorio de estadística tiene como objetivo desarrollar los métodos de inferencia estadística. Este es uno de los temas más enseñados, pero a la vez, es el peor comprendido y utilizado. Entendiendo que las asignaturas de estadística requieren un avance progresivo, desde lo más simple a lo más complejo, se requiere que el estudiante entienda con claridad que es y para qué sirve la inferencia estadística.

Entendamos el término informal como parte del conocimiento ordinario del estudiante, es decir, el conocimiento que poseen los estudiantes antes de ingresar al salón, fuera de clases, en la vida cotidiana. Teniendo presente la importancia de los saberes previos, ya que estos son el punto de partida para lograr el aprendizaje (Rodríguez, 2012).

García (2013) explica que la Inferencia Estadística Informal es un tipo de razonamiento entre el análisis exploratorio de datos y la inferencia estadística formal, y señala que el término informal se refiere a la posibilidad de utilizarlo fuera de los procedimientos formales. Estrella (2017) refiere que la ISI consiste en realizar inferencias sobre la población basada en muestras aleatorias de datos que los estudiantes hayan recolectado. A través del análisis de estos datos se realizará la generalización mediante un lenguaje probabilístico, enfatizando cierta incertidumbre sobre esta inferencia.

El razonamiento que fomenta la ISI posee una 
gran importancia debido a que los estudiantes utilizan su conocimiento informal para argumentar el apoyo a las inferencias realizadas a partir de las muestras (Rodríguez, 2012). García (2013) explica que el análisis del razonamiento inferencial informal de los estudiantes se lleva a cabo considerando cuatro componentes, los cuales son: 1 . Conclusión más allá de los datos. Comprende hacer juicios, afirmaciones o predicciones acerca de las poblaciones a partir de los datos recolectados de la muestra, pero sin las técnicas y procedimientos formales. Hacer estimaciones de fenómenos inciertos; 2. Uso de los datos como evidencia. La articulación de los argumentos para realizar los juicios, afirmaciones o predicciones sobre la población. Basar las conclusiones en los patrones de los datos disponibles. Centrarse en la generalización de resultados para lograr la comprensión en los estudiantes; 3. Uso de lenguaje probabilístico que exprese incertidumbre de la conclusión. Hay que recordar que en la estadística formal se elaboran afirmaciones que no tienen certeza absoluta. Entendiendo que una predicción es solo una estimación; y 4. Utilizar e integrar el conocimiento previo (conocimiento formal e informal). Las inferencias se elaboran a partir de los datos disponibles, y además debe ir acompañada de una teoría (científica o personal) que permita realizar e interpretar dicha generalización.

En los trabajos de García (2013) y Rodríguez (2012) se exponen casos sobre el empleo de la inferencia estadística informal en clases.

\section{Modelos culturales en la enseñanza de la estadística.}

Witusba (2014) afirma que un modelo didáctico no surge de la nada, sino que son producto de las prácticas docentes, su organización didáctica y los esfuerzos colaborativos. Los modelos culturales se refieren a modelos didácticos, principalmente utilizados en el nivel escolar, pero cabe señalar que es posible aplicarlos en niveles superiores, pero como habrá deducido el lector, principalmente con los modelos culturales se atiende al nivel de la alfabetización estadística. Asimismo, explica que los modelos culturales se centran en aquello que la cultura ha validado, lo que significa que "aquello que se aprende se replica". Es decir que tienen un componente orientado hacia lo pragmático. En estos modelos, se considera la división entre: modelos interpretativos (MI), que se caracterizan por comprender la entrega de la información ya procesada para ser leída e interpretada por el estudiante; y modelos operativos (MO), que se caracterizan por establecer el análisis exploratorio y operativo de los datos a través del uso de estadígrafos de tendencia central, para describir la información; y el modelo productivo investigativo (MPI), que se caracteriza por generar información de carácter original, utilizando la metodología de investigación. En la Tabla 1 se muestra un resumen de los tres modelos explicados por Witusba (2014).

\section{Tabla 1}

Modelos culturales en la enseñanza de estadística

\begin{tabular}{|l|l|l|l|}
\hline & Modelos interpretativos & Modelos Operativos & $\begin{array}{l}\text { Modelos productivos } \\
\text { investigativos }\end{array}$ \\
\hline $\begin{array}{l}\text { Visión de } \\
\text { aprendizaje }\end{array}$ & $\begin{array}{l}\text { La visualización de la información } \\
\text { propicia un movimiento reflexivo. }\end{array}$ & $\begin{array}{l}\text { La práctica del análisis } \\
\text { estadístico promueve el } \\
\text { aprendizaje. }\end{array}$ & $\begin{array}{l}\text { Integrar la estadística como parte } \\
\text { del proceso investigativo. }\end{array}$ \\
\hline Objetivo & $\begin{array}{l}\text { Interpretar y comparar infor- } \\
\text { mación a través de gráficos y } \\
\text { emplearlos para hipotetizar rela- } \\
\text { ciones. }\end{array}$ & $\begin{array}{l}\text { Comprender los procedimientos } \\
\text { estadísticos a través de } \\
\text { la elaboración tablas de } \\
\text { frecuencias y la obtención de } \\
\text { estadígrafos. }\end{array}$ & $\begin{array}{l}\text { Elaborar y ejecutar proyectos } \\
\text { de investigación que fomenten } \\
\text { el interés del estudiante para } \\
\text { responder preguntas a través del } \\
\text { uso de estadística. }\end{array}$ \\
\hline $\begin{array}{l}\text { Material } \\
\text { empleado } \\
\text { y acciones } \\
\text { requeridas }\end{array}$ & $\begin{array}{l}\text { Boletas de pago } \\
\text { Información de productos de con- } \\
\text { sumo } \\
\text { Situaciones de carácter vivencial } \\
\text { (peso, talla, etc) }\end{array}$ & $\begin{array}{l}\text { Entrega de datos brutos. } \\
\text { Recolección de datos brutos. }\end{array}$ & $\begin{array}{l}\text { Elaboración de un proyecto de } \\
\text { investigación. } \\
\text { Planificación del trabajo. } \\
\text { Recolección de datos. }\end{array}$ \\
\hline
\end{tabular}




\section{Ambiente de aprendizaje para el razonamiento estadístico (SRLE).}

Estrella (2017) afirma que el Ambiente de Aprendizaje para el Razonamiento Estadístico (Statistical Reasoning Learning Environment, SRLE) consiste en un ambiente que promueve razonar estadísticamente y con la intención de que los estudiantes logren una comprensión profunda de la estadística. Ben-Zvi (2011) explica que este modelo se basa en la teoría de aprendizaje y enseñanza socio-constructivista y se sustenta en seis principios pedagógicos: Enfocado en el desarrollo de ideas estadísticas centrales, en lugar de las técnicas, procedimientos; Usa datos reales y motivadores logrando incrementar el interés de los estudiantes, que tienen que hacer y probar conjeturas; Usa actividades en clase para apoyar el desarrollo del razonamiento de los estudiantes; Integra el uso de herramientas tecnológicas apropiadas que permitan a los estudiantes probar sus conjeturas, explorar y analizar datos y desarrollar su razonamiento estadístico; Promueve un discurso en clases que argumente a través de términos estadísticos dirigidos a las ideas estadísticas significativas; y Utiliza el diagnóstico para aprender lo que los estudiantes saben y monitorear el desarrollo de su aprendizaje estadístico para evaluar los planes de instrucción y su avance.

Este modelo se le denomina entorno de aprendizaje debido a que se trata de una combinación interactiva de materiales de texto, actividades de clase, normas y cultura, discusión, tecnología, acercamiento docente y evaluación. La Figura 2 presenta los seis elementos que comprende el SRLE mostradas en el trabajo de Ben-Zvi (2011).

En el trabajo de Ben-Zvi (2011) se pueden encontrar dos ejemplos de la aplicación del modelo en situaciones reales: el primero para ambientes universitarios y el segundo para ambientes escolares.

\section{Aprendizaje gamificado en la enseñanza estadística.}

La gamificación se basa en el uso de elementos de videojuegos usados en contextos diferentes al mismo juego; se han producido intentos de gamificar actividades en sectores diversos, como la educación, empresas, recursos humanos, etc. (Ortíz-Colón, Jordán \& Agredal, 2018). Landers (2015) propone una teoría del aprendizaje gamificado que sustenta cinco proposiciones principales: El contenido instruccional influye en los resultados del aprendizaje y en los comportamientos/actitudes, los comportamientos/actitudes influyen en el aprendizaje, las características de los juegos influyen en el cambio de comportamientos/actitudes, los elementos de los juegos afectan los comportamientos/ actitudes que moderan la efectividad de las instrucciones y la relación entre los elementos del juego y los resultados del aprendizaje, la cual

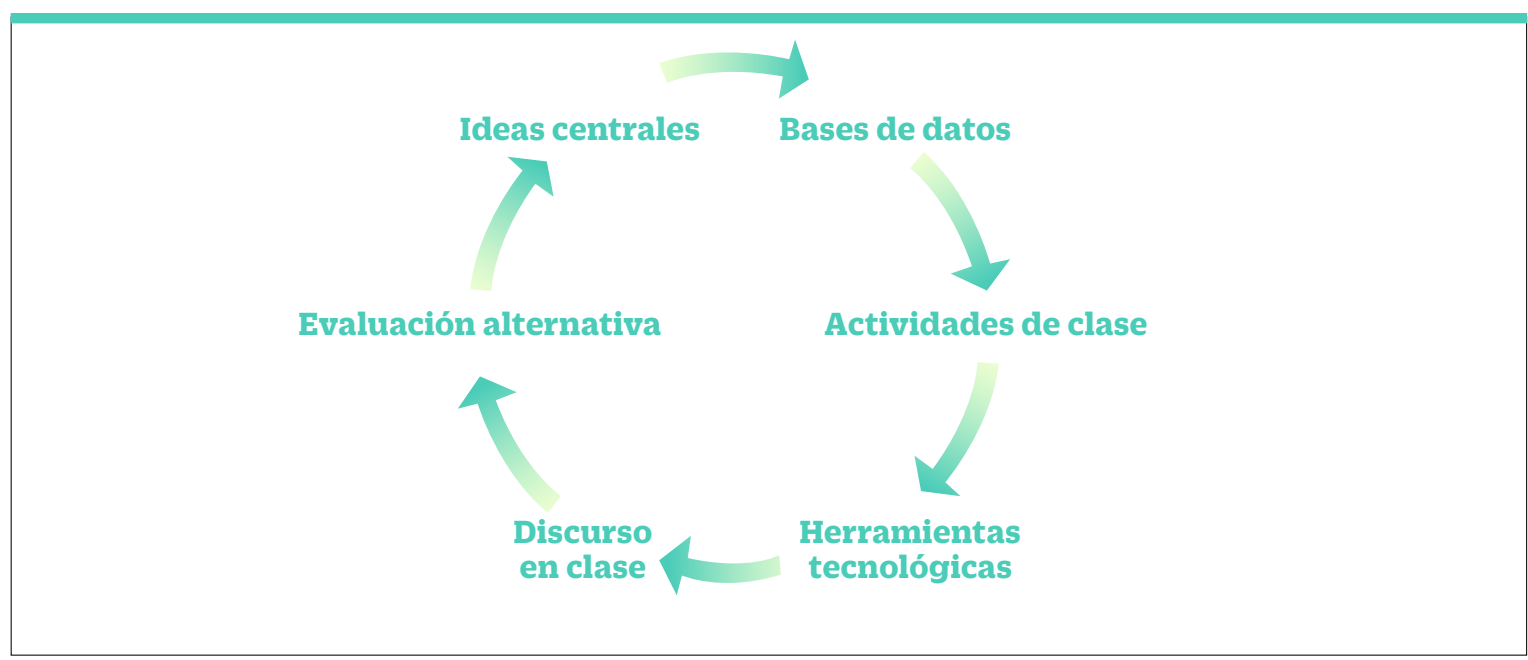

Figura 2. Los seis elementos del SRLE 


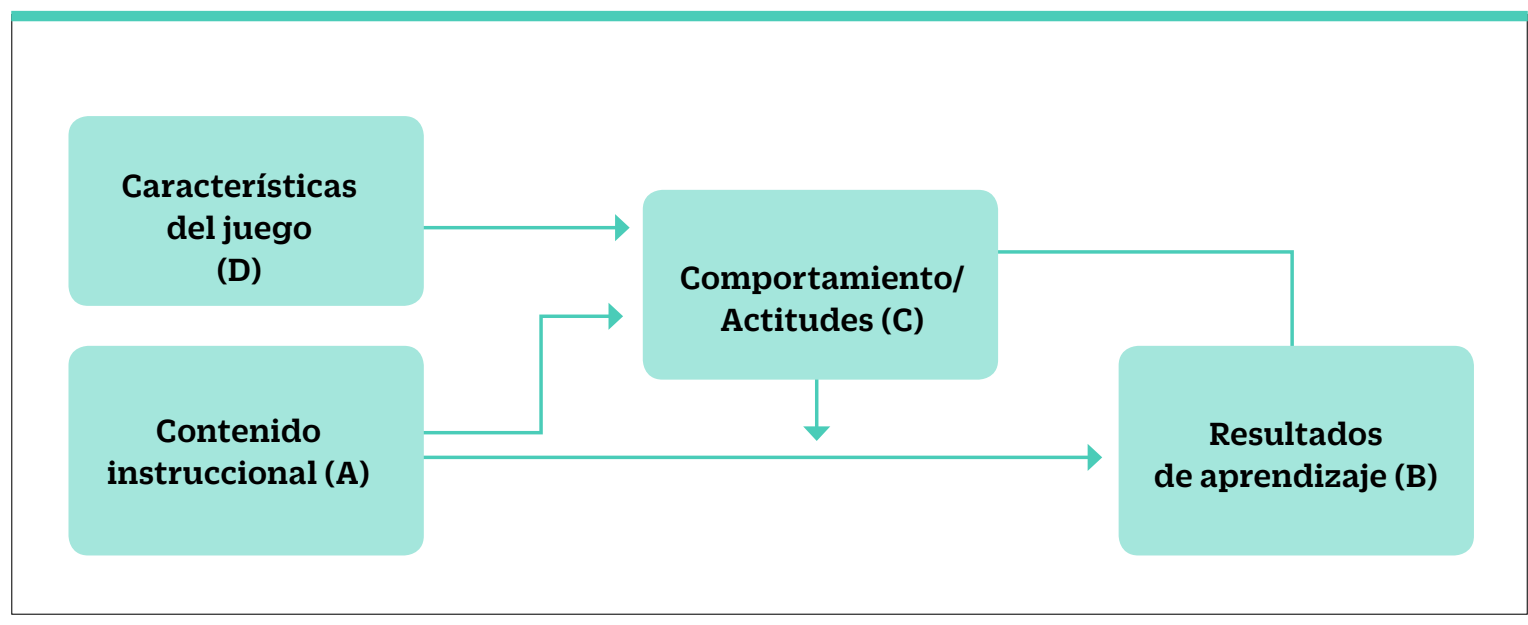

Figura 3. Teoría del aprendizaje gamificado de Landers (2015)

es mediada por los comportamientos/actitudes. La Figura 3 presenta un diagrama elaborado por Landers (2015) que resume su teoría.

Todavía no existe mucha información disponible sobre la gamificación en cursos de estadística, sin embargo, los resultados se muestran prometedores. Boyle et al. (2014) realizaron una revisión de literatura al respecto. Para la búsqueda de información se utilizaron criterios de búsqueda que estuvieran relacionados con los juegos, simulaciones y animaciones, gamificación, e-learning, y aprendizaje asistido por la computadora, encontrando 26 estudios de las Bases de datos ERIC, EBSCO (PsycINFO, SocINDEX, Library, Information Science and Technology Abstract, CINAHL), ASSIA, respecto a cómo la utilización de juegos digitales, simulaciones y animaciones lograban objetivos de aprendizaje en cursos de metodología y estadística, concluyendo que existen razones para ser optimista. Las aproximaciones basadas en juegos pueden ser efectivas para el aprendizaje en esta área. En un estudio reciente, Smith (2017) utilizó la teoría de Landers (2015) para investigar como la instrucción mediante módulos gamificados en un curso de estadística afecta el aprendizaje y las actitudes hacia la estadística en estudiantes de pre-grado, encontrando que los estudiantes que siguieron la instrucción gamificada, tuvieron cambios positivos en sus actitudes y en su aprendizaje en estadística. Este resultado aporta evidencia inicial sobre la efecti- vidad de una aproximación mediante la gamificación en cursos de estadística.

Se recomienda al lector que se dirija a los trabajos de Landers (2015), Landers, Auer, Collmus y Armstrong (2018), Smith (2017) y Ortíz-Colón et al. (2018) para conocer más sobre el aprendizaje gamificado. En el trabajo de Barreal, Hernández y Jannes (2016) puede encontrar una propuesta detallada de un programa gamificado.

\section{Discusión}

Vivimos en un momento único en la historia de la humanidad, caracterizado por una revolución tecnológica equiparable solo a la revolución industrial en lo concerniente al impacto en la vida del hombre. Esta revolución tecnológica ha establecido nuevas formas de relacionarnos y ha elevado exponencialmente la información que tenemos al alcance, la cual ahora es infinita y sigue creciendo a un ritmo acelerado día a día.

Este crecimiento acelerado de información se ha vuelto parte fundamental del desarrollo de las sociedades. Acosta y Mejía (2017) explican que "la relación entre el desarrollo de un país y el grado en que su sistema estadístico produce estadísticas completas y fiables es clara, porque esta información es necesaria para la toma de decisiones 
acertadas de tipo económico, social y político" (p.3). Por lo mencionado, es necesario formar a los nuevos ciudadanos con competencias pertinentes para entender y analizar datos, beneficiándose de las oportunidades que conlleva trabajar con este tipo de información; especialmente en el contexto de la formación de escolares y profesionales, quienes deben tener las herramientas y habilidades necesarias para poder aprovechar la gran cantidad de información económica, educativa, social, cultural y política de su alrededor (Rodríguez-Alveal, 2017), logrando que la educación estadística sea un motor de desarrollo (Batanero, 2018), tanto social como individual.

La educación estadística en niveles superiores se presenta como un reto y como una oportunidad. Un reto debido a la existencia de una problemática particular en sus procesos de enseñanza aprendizaje, siendo esta problemática más marcada en este curso que en otros. Esta problemática se presenta en un gran número de salones universitarios, y es especialmente notoria, en carreras que no están muy próximas a las matemáticas (Rodríguez, 2011). Dentro de las características que conlleva se pueden hablar de niveles altos de ansiedad, actitudes desfavorables, falta de motivación e interés por parte de los estudiantes, frustraciones del docente al no poder mejorar los procesos y hasta se podría hablar de miedo hacia la asignatura (Behar \& Grima, 2004; Molina et al., 2011). Es especialmente interesante, considerar el rol que juegan las actitudes, ya que estas comprenden la forma en que una persona responde hacia algo o alguien, para este caso se trata sobre como el sujeto responde hacia la estadística, y que lo predispone al individuo a actuar acorde a las creencias, pensamientos y emociones frente al objeto de la actitud.

Si bien el estudiante puede entender a un nivel cognitivo que la estadística es importante para hacer investigaciones científicas y el desarrollo de la ciencia, a un nivel afectivo puede presentar reacciones aversivas hacia el curso, lo que genera un conflicto en lo que piensa y en cómo se siente, es en este conflicto que el estudiante se predispone a no darle importancia y no esforzarse ni interesarse (nivel conductual) por su aprendizaje y aplicación. En cualquier curso de estadística, sin importar la carrera, uno de los objetivos que se debe trazar el docente implica la modificación de actitudes hacia la estadística, ya que las actitudes positivas se espera que mejoren su proceso de aprendizaje e incrementen su rendimiento en los exámenes del curso, e incluso el implementar sus habilidades estadísticas en la vida profesional (Kiekkas et al., 2015).

La asignatura de estadística se presenta como un reto por las dificultades inherentes que comprende un curso de esta naturaleza, también es una oportunidad para el docente de fortalecer sus competencias y crear estrategias creativas que logren cambios significativos y que impulsen el desarrollo del pensamiento estadístico, lo que implica que el docente debe ser un profesional en constante preparación para la enseñanza de la estadística. Si este se inclina por modelos tradicionales de enseñanza, que se centran excesivamente en los contenidos y la aplicación de los procedimientos matemáticos sin enfatizar en la utilidad ni la interpretación de resultados, posiblemente tendrá un efecto contraproducente en los estudiantes que, en vez de atraerlos hacia esta ciencia, estos tendrán reacciones más negativas en comparación a como ingresaron el primer día a sus clases de estadística. Blanco (2018) explica que la estadística actual es muy distinta a la estadística del siglo pasado, ya que, debido a la gran cantidad de datos, sus métodos han ido cambiando y expandiéndose, lo que ha exigido nuevas formas de procesar la información, modificando como se piensa y como se promueve el aprendizaje a partir de los datos. Estos cambios han promovido un gran interés en el estudio de la didáctica de la estadística.

Actualmente, los cursos de estadística, en cualquier carrera universitaria, deben planificarse apuntando a desarrollar el pensamiento estadístico en sus estudiantes. Lo cual es un escenario ideal, pero muchos de sus estudiantes se encuentran en niveles inferiores o en el peor de los casos aún no han alcanzado niveles inferiores como la alfabetización o el razonamiento estadístico, y es en la universidad donde se tiene que suplir algunas deficiencias de la educación estadística en niveles básicos de educación para alcanzar el nivel cognitivo superior, el pensamiento estadístico. La utilización de estos niveles cognitivos para su enseñanza ha favorecido de forma importante 
para establecer hitos relevantes en el proceso, lo que Behar y Grima (2004) expresan de la siguiente forma:

Suena razonable, empezar por discutir y reflexionar sobre lo que se pretende lograr con nuestro curso de estadística, pues teniendo razonablemente claro nuestro punto de llegada y conociendo nuestro punto de partida, tendremos mayores probabilidades de éxito en el trazado de una ruta que intente conectar dichos puntos (p.86).

La oportunidad que ofrece enseñar el curso de estadística comprende que el docente asuma un papel activo como investigador sobre su didáctica, para que conozca los modelos didácticos actuales, evaluarlos y elegir aquel o aquellos que se ajusten a las necesidades del curso. De los modelos didácticos presentados, se recomienda revisar con mayor detenimiento (al menos inicialmente), el propuesto por la ASA (2016) que se considera como uno de los más importantes en la enseñanza de la estadística. El cumplimiento de las metas sugeridas en dicho informe tendrá repercusiones en el corto plazo, y sumado a la aplicación pertinente de los otros modelos, logrará desarrollar el pensamiento estadístico en sus estudiantes.

Dentro de las recomendaciones, limitaciones y proyecciones producto de la presente revisión, se podría enlistar tres grandes bloques que engloban algunos problemas en el estudio y la aplicación de la didáctica de la estadística en contexto universitario. El primero comprende el estudio de las actitudes hacia la estadística, que está cobrando un papel muy importante en los procesos de enseñanza aprendizaje de la asignatura. El tema de evaluación de actitudes está creciendo de forma importante, tanto en aspectos metodológicos, la creación y validación de instrumentos, y aspectos de la investigación aplicada, la evaluación del impacto de programas para la modificación de actitudes en cursos de estadística. El segundo recae en la evaluación de las aptitudes del estudiante. Resulta importante realizar estudios sobre la capacidad del estudiante universitario para desarrollar operaciones estadísticas, ya sean manuales o con apoyo de la tecnología, y determinar el nivel cognitivo (alfabetización, razonamiento o pensamiento estadístico) con el que llegan a la asignatura. Ya que un estudiante podría estar motivado para aprenderla (actitud favorable), pero no tener una formación adecuada previa, lo que podría afectar el logro de la competencia en la asignatura. El tercero se dirige hacia la elección del modelo didáctico por parte del docente, por lo que desde un inicio debe identificar cual es el punto de partida y apuntar a una meta (el punto de llegada), que implica evaluar a priori las actitudes y aptitudes de los estudiantes y el contexto de la clase, para lo cual debe considerar el número de estudiantes en el aula, el acceso a tecnología, los intereses de los estudiantes, la relación de los contenidos con el programa profesional, los conocimientos y actitudes del propio docente hacia la estadística, entre otros factores que puedan tener influencia en el proceso. Teniendo la información recopilada, proponer acciones basadas en la evidencia que puedan ser evaluadas y expuestas para enriquecer esta línea de investigación.

Como reflexión final, el lector ha podido apreciar aspectos generales sobre la didáctica de la estadística, comprendiendo desde la problemática en la enseñanza, los niveles cognitivos en su aprendizaje y la presentación de modelos didácticos para aplicar en sus clases, por lo que ahora tiene una idea de cuánto trabajo se requiere en este campo tan apasionante. El investigador educativo debe comprender que la estadística no es solo una herramienta para el procesamiento de datos, sino que es una forma inteligente de ver el mundo, que propicia la toma de decisiones basadas en la evidencia y la resolución de problemas de la vida cotidiana en beneficio individual, como social. 


\section{Referencias}

Acosta, J. A. \& Mejía, D. E. (2017). Actitudes hacia la estadística de los estudiantes de pregrado de la Universidad Nacional Pedro Ruiz Gallo. Revista Científica Institucional Tzhoecoen, 9(3). https://doi.org/10.26495/ rtzh179.323428

Alsina, A. (2017). Contextos y propuestas para la enseñanza de la estadística y la probabilidad en Educación Infantil: un itinerario didáctico. Épsilon - Revista de Educación Matemática, 34(95), 25-48.

American Statistical Association (2016). Guidelines for as sessment and instruction in statistics education (GAISE) College Report. Recuperado de http://www. amstat.org/education/gaise

Andrade, L., Fernández, F. \& Álvarez, I. (2017). Panorama de la investigación en educación estadística desde tesis doctorales 2000-2014. Tecné Episteme $Y$ Didaxis: TED, (41), 87-107. https://doi. org/10.17227/01203916.6039

Aparicio, A. S. \& Bazán, J. L. (2006). Actitud y rendimiento en estadística en profesores peruanos. Acta Latinoamericana de Matemática Educativa, 19, 644-650. Recuperado de https://goo.gl/hPLqkW

Barreal, J., Hernández, A. \& Jannes, G. (2016). La gamificación como herramienta didáctica aplicada a métodos estadísticos en el proceso de aprendizaje de las enseñanzas turísticas. Recuperado de https://aecit. org/files/congress/20/papers/360.pdf

Batanero, C. (2001). Didáctica de la estadística. Granada: Grupo de investigación en educación estadística. Recuperado de https://goo.gl/3py5DK

Batanero, C. (2018). Treinta años de Investigación Didáctica sobre el análisis inferencial de datos. En A. Avila (Coord.), Rutas de la Educación Matemática. México. Sociedad Mexicana de Investigación y Divulgación de la Educación Matemática.

Behar, R, \& Grima, P. (2004). La estadística en la educación superior ¿Formamos pensamiento estadístico?. Ingeniería y Competitividad, 5(2), 84-90. DOI: https:// doi.org/10.25100/iyc.v5i2.2299

Behar, R., Grima, P., Ojeda, M. M. \& Cruz, C. (2013). Educación estadística en cursos introductorios a nivel universitario: algunas reflexiones. En A. Salcedo (Ed.), Educación Estadística en América Latina Tendencias y Perspectivas (pp. 343-360). Caracas: Programa de cooperación interfacultades de la Universidad Central de Venezuela. Recuperado de https://goo.gl/n6RggK
Ben-Zvi, D. (2011). Statistical Reasoning learning environment. Em Teia - Revista de Educacao Matemática e Tecnológica Iberoamericana, 2(2). Recuperado de https://goo.gl/kLAAT4

Ben-Zvi, D. \& Garfield, J. (2004). Statistical literacy, reasoning, and thinking: Goals, definitions, and challenges. En D. Ben-Zvi y J. Garfield (Eds.), The challenge of developing statistical literacy, reasoning and thinking (pp. 3-15). New York: Springer. Recuperado de https://goo.gl/SZULCv. https://doi.org/10.1007/1 4020-2278-6

Bertorello, N., Albrecht, G. \& Tauber, L. (Agosto, 2010). Re visión de estudios que analizan las actitudes y ansiedad respecto de la estadística y de la ciencia. Trabajo presentado en el III REPEM - Memorias. Recuperado de https://goo.gl/aeuZs5

Blanco, A. (2008). Una revisión crítica de la investigación sobre las actitudes de los estudiantes universitarios hacia la estadística. Revista Complutense de Educación, 19(2), 311-330. Recuperado de https:// goo.gl/Yu1vjK

Blanco, A. (2018). Directrices y recursos para la innovación en la enseñanza de la Estadística en la Universidad: Una revisión documental. Revista de Docencia Universitaria, 16(1), 251-267. https://doi.org/10.4995/ redu.2018.9372

Boyle, E. A., MacArthur, E. W., Connolly, T. M., Hainey, T., Manea, M., Karki, A. \& van Rosmalen, P. (2014). A narrative literatura review of games, animations and simulations to teach research methods and statistics. Computers \& Education, 74. https://doi. org/10.1016/j.compedu.2014.01.004

Burga, A. (2010). Valoración de objetivos en la enseñanza de la estadística en estudiantes de psicología. Persona, 13, 111-124. Recuperado de https://goo.gl/Vzi6ge. https://doi.org/10.26439/persona2010.n013.267

Campos, C. R. (Agosto, 2016). La educación estadística y la educación crítica. Trabajo presentado en 2do Encuentro Colombiano de Educación Estocástica. http://doi.org/10.13140/RG.2.2.21916.08324

Comas, C., Martins, J. A., Nascimento, M. M. \& Estrada, A. (2017). Estudio de las actitudes hacia la estadística en estudiantes de psicología. Bolema, Rio Claro (SP), 31(57), 479-496. http://doi.org/10.1590/1980-4415v31n57a23

Dempster, M. \& McCorry, N. K. (2009). The role of previous experience and attitudes towards statistics in statistics asesssment outcomes among undergraduate psychology students. Journal of Statistics Edu- 
cation, 17(2). https://doi.org/10.1080/10691898.200 9.11889515

Díaz-Reissner, C. V. \& Quintana-Molinas, M. E. (2018). Actitud hacia la estadística en estudiantes de odontología. Odontología SanMarquina, 21(3), 173-179. http://doi. org/10.15381/os.v21i3.15130

Dillon, K. M. (1992). Statisticophobia. Teaching of Psychology, 9(2), 117. Recuperado de https://goo.gl/6pqG51. https://doi.org/10.15381/os.v21i3.15130

Estrada, A. (2002). Análisis de las actitudes y conocimientos estadísticos elementales en la formación del profesorado. Tesis de doctorado. Universidad Autónoma de Barcelona, Barcelona, España.

Estrella, S. (2017). Enseñar estadística para alfabetizar estadísticamente y desarrollar el razonamiento estadístico. En: Salcedo, A. (Comp.). Alternativas Pedagógicas para la Educación Matemática del Siglo XXI, (173 - 194). Caracas: Centro de Investigaciones Educativas, Escuela de Educación. Universidad Central de Venezuela. Recuperado de https://goo.gl/tUFF5Y

García, V. N. (2013). Inferencias estadísticas informales en estudiantes mexicanos. En J. M. Contreras, G. R. Cañadas, M. M. Gea \& P. Arteaga (Eds.), Actas de las Jornadas Virtuales en Didáctica de la Estadística, Probabilidad y Combinatoria (pp. 343-357). Granada: Departamento de didáctica de la matemática de la Universidad de Granada. Recuperado de https:// goo.gl/eWjo57

García-Martínez, J. A., Fallas-Vargas, M. A. \& Romero-Hernández, A. (2015). Las actitudes hacia la estadística del estudiantado de orientación. Revista Electrónica Educare, 19(1), 25-41. http://doi.org/10.15359/ ree.19-1.2

Kiekkas, P., Panagiotarou, A., Malja, A., Tahirai, D., Zykai, R., Bakalis, N. \& Stefanopoulos, N. (2015). Nursing students' attitudes towards statistics: effect of a biostatistics course and association with examination performance. Nurse Education Today. 35(12), 1283 1288. https://doi.org/10.1016/j.nedt.2015.07.005

Landers, R. N. (2015). Developing a theory of gamified learning: linking serious games and gamification of learning. Simulation \& Gaming, 45(6), 752-768. https://doi.org/10.1177/1046878114563660

Landers, R. N., Auer, E. M., Collmus, A. B. \& Armstrong, M. B. (2018). Gamification science, its history and future: definitions and a research agenda. Simulation \& Gaming,1-23.https://doi.org/10.1177/1046878118774385

Lesser, L. M. (Julio, 2011). Making statistics memorable: new mnemonics and motivations. Trabajo presentado en Joint Statistical Meetings, Miami Beach, Florida. Recuperado de https://goo.gl/Mjmvg4

Mason, S. E. \& Reid, E. M. (2018). The relationship between anxiety and performance in a statistical class. Mathematics for Aplications, 7, 63-70. http://doi. org/10.13164/ma.2018.06

Molina, J., Rodrigo, M. \& Bonavia, T. (2011). La docencia de estadística en el grado de psicología: una experiencia de colaboración interdisciplinar con la asignatura de psicología social del trabajo. @Tic Revista D 'innovació Educativa, 6, 21-29. Recuperado de https://goo.gl/ CYNe3Q. https://doi.org/10.7203/attic.6.287

Onwuegbuzie, A. J. (2004). Academic procrastination and statistics anxiety. Assessment \& Evaluation In Higher Education, 29(1), 3-19. https://doi. org/10.1080/0260293042000160384

Orozco, G. H., Sosa, M. R. \& Martínez, F. (2018). Modelos didácticos en la educación superior: una realidad que se puede cambiar. Profesorado Revista de Currículum y Formación del Profesorado, 22(2), 447-469. http://doi.org/10.30827/profesorado.v22i2.7732

Ortiz-Colón, A. M., Jordán, J. \& Agredal, M. (2018). Gamificación en educación: una panorámica sobre el estado de cuestión. Educacao e Pesquisa, 44. http://doi. org/10.1590/s1678-4634201844173773

Paechter, M., Macher, D., Martskvishvili, K., Wimmer, S. \& Papousek, I. (2017). Mathematics anxiety and statistics anxiety. Shared but also unshared components and antagonistic contributions to performance in statistics. Frontiers in Psychology, 8. https://doi. org/10.3389/fpsyg.2017.01196

Pérez, C. G. (2010). La estadística como herramienta en la investigación psicológica un estudio exploratorio. Trabajo final de máster no publicado, Universidad Pedagógica Nacional Unidad Ajusco México. Recuperado de https://goo.gl/BebJxx

Pérez, L. E., Aparicio, A. S., Bazán, J. L. \& Abdounur, O. J. (2015). Actitudes hacia la estadística en estudiantes universitarios de Colombia. Educación Matemática, 27(3), 111-149.

Régnier, J. C. \& Kuznetsova (2014). Teaching of statistics: formation of statistical reasoning. Procedia - Social and Behavioral Science, 154(28), 99-103. https://doi. org/10.1016/j.sbspro.2014.10.119

Rodríguez, N. (2011). Actitudes de los estudiantes universitarios hacia la estadística. Interdisciplinaria, 28(2), 199-205. Recuperado de https://goo.gl/6SjUWm

Rodríguez, M. I. (2012). Inferencia informal: del análisis de 
los datos a la inferencia estadística. Revista de educación matemática. Recuperado de https://goo.gl/ zWLh4Y

Rodríguez-Alveal, F. E. (2017). Alfabetización estadístico en profesores de distintos niveles formativos. Educacao \& Realidade, 42(4), 1459-1477. http://doi. org/10.1590/2175-623662610

Ruiz, N. (2015). La enseñanza de la estadística en la educación primaria en América Latina. REICE. Revista Iberoamericana sobre Calidad, Eficacia y Cambio en Educación, 13(1), 103-121.

Ruiz de Miguel, C. (2015). Actitudes hacia la estadística de los alumnos del grado en pedagogía, educación social, y maestro de educación infantil y maestro de educación primaria de la UCM. Educación XXI: Revista de la Facultad de Educación, 18(2), 351-374. https://doi.org/10.5944/educxx1.14608

Salinas, J. \& Mayén, S. (2016). Estudio exploratorio de las actitudes hacia la estadística en estudiantes mexicanos de bachillerato. Avances de Investigación en Educación Matemática, 10, 73-90. https://doi. org/10.35763/aiem.vOi10.130

Sánchez, A. G. \& Berenguer, I. A. (2014). Un sistema de procedimientos didácticos para potenciar la formación del pensamiento estadístico en el nivel universitario. Revista Orbita Pedagógica, 1(3), 41-54. Recuperado de https://goo.gl/t8uXZB

Sanoja, J. E. \& Ortíz, J. (2013). El conocimiento didáctico del contenido estadístico del maestro. En A. Salcedo (Ed.), Educación Estadística en América Latina Tendencias y Perspectivas (pp.125-150). Caracas: Programa de cooperación interfactultades de la Universidad Central de Venezuela. Recuperado de https://goo.gl/n6RggK

Schield, M. (2017). GAISE 2016 promotes statistical literacy. Statistical Education Research Journal, 16(1), 46-50. Recuperado de https://goo.gl/igaQCD

Smith, T. (2017). Gamified modules for an introductory statistics course and their impact on attitudes and learning. Simulation \& Gaming, 48(6), 832-854. ht- tps://doi.org/10.1177/1046878117731888

Sowey, E. R. (1995). Teaching statistics: making it memorable. Journal of Statistical Education, 3:2. https://doi. org/10.1080/10691898.1995.11910487

Tarazona, E., Bazán, J. L. \& Aparicio, A. S. (2013). Actitudes hacia la estadística en universitarios peruanos de mediana edad. Revista Digital de Investigación en Docencia Universitaria, 7(1), 57-76. https://doi. org/10.19083/ridu.7.187

Thomas, L. (2007). Understanding statistics and research through metaphors. Journal of Social Service Research, 34(2), 1-14. https://doi.org/10.1300/ J079v34n02_01

Toapanta-Toapanta, G. M., Pérez-Narváez, M. V. \& Lema-Yungan, J. G. (2018). Las competencias para el aprendizaje de la estadística en los estudiantes de educación superior. Roca. Revista Científico-Educacional de la Provincia de Granma, 14(1). Recuperado de https://revistas.udg.co.cu/index.php/roca/article/ view/262/408

Vázquez, L. P., Aguilar, E. C., Chávez, M., Bony, P. L. \& Montes de Oca, A. (2016). La enseñanza problémica como alternativa didáctica en el tema de Estadísticas Sanitarias para la asignatura Metodología de la Investigación y Estadística. Revista de Información Cien tífica, 95(6), 985-993. Recuperado de https://dialnet. unirioja.es/servlet/articulo?codigo $=6027458$

Wild, C. J. \& Pfannkuch, M. (1999). Statistical thinking in empirical enquiry. International Statistical Review, 67, 223-265. Recuperado de https://goo.gl/f4Mjsm. https://doi.org/10.1111/j.1751-5823.1999.tb00442.x

Witusba, L. (2014). Didáctica de la estadística: modelos culturales en la enseñanza de la estadística. Sabvia Revista de Educación. Recuperado de https://goo. gl/PC7g2p

Zapata, L. (2011). ¿Cómo contribuir a la alfabetización estadística?. Revista Virtual Universidad Católica del Norte, 33, 234-247. Recuperado de https://goo.gl/ QXRrGg es un artículo de acceso abierto, distribuido bajo los términos de la LicenciaCreativeCommons Atribución-CompartirIgual 4.0 Internacional.(http://creativecommons.org/licenses/by-sa/4.0/), que permite el uso no comercial, distribución y reproducción en cualquier medio, siempre que la obra original sea debidamente citada. 